\title{
How to Estimate the Value of Service Reliability Improvements
}

\author{
Michael J. Sullivan, Chairman, FSC, Matthew G. Mercurio, Senior Consultant, FSC, Josh A. \\ Schellenberg, Senior Analyst, FSC, and Joseph H. Eto, Staff Scientist, LBNL
}

\begin{abstract}
A robust methodology for estimating the value of service reliability improvements is presented. Although econometric models for estimating value of service (interruption costs) have been established and widely accepted, analysts often resort to applying relatively crude interruption cost estimation techniques in assessing the economic impacts of transmission and distribution investments. This paper first shows how the use of these techniques can substantially impact the estimated value of service improvements. A simple yet robust methodology that does not rely heavily on simplifying assumptions is presented. When a smart grid investment is proposed, reliability improvement is one of the most frequently cited benefits. Using the best methodology for estimating the value of this benefit is imperative. By providing directions on how to implement this methodology, this paper sends a practical, usable message to the industry.
\end{abstract}

Index Terms--Economics, education, planning, power distribution reliability, power system reliability, power transmission reliability, reliability, reliability estimation, statistics, technology planning

\section{INTRODUCTION}

$\mathrm{R}$ eliability improvement is one of the most frequently cited justifications for investments in smart grid technology. Although reliability undoubtedly improves under most smart grid investments, the value of the reduction in outage frequency and/or duration is difficult to calculate. This difficulty leads many analysts to make simplifying assumptions. In a recent report by Freeman, Sullivan \& Co. (FSC) and Lawrence Berkeley National Laboratory (LBNL), the authors propose a methodology for calculating the value of reliability improvements that does not as heavily rely on simplifying assumptions. This methodology has been proven to provide more reliable estimates, but has not usurped other methodologies because it is more complicated. This paper deals with that complication to guide analysts on how to apply outage cost estimates to a smart grid investment opportunity.

This work was supported in part by the U.S. Department of Energy under Contract No. DE-AC02-05CH11231.

Michael J. Sullivan is with Freeman, Sullivan \& Co., San Francisco, CA 94115 USA (e-mail:michaelsullivan@fscgroup.com).

Matthew G. Mercurio is with Freeman, Sullivan \& Co., San Francisco, CA 94115 USA (e-mail:matthewmercurio@fscgroup.com).

Josh A. Schellenberg is with Freeman, Sullivan \& Co., San Francisco, CA 94115 USA (e-mail:joshschellenberg@fscgroup.com).

Joseph H. Eto is with Lawrence Berkeley National Laboratory, Berkeley, CA 94720 USA (e-mail: jheto@lbl.gov).

\section{Estimating Outage Costs}

Starting in the mid-1980s, utilities in the US conducted a number of customer outage cost studies using slightly different survey methods and procedures. Survey-based methods have become the most widely used approach and are generally preferred over other measurement protocols because they can be used to obtain outage costs for a wide variety of reliability and power quality conditions not observable using other techniques. ${ }^{1}$ Commercial customers are asked about the value of lost production, other outage related costs, and outage related savings, after taking into account their ability to make up for any lost production. For residential customers, the vast share of outage impacts are not directly observable economic costs and, as a result, they are typically asked about their willingness to pay to avoid outages with specific characteristics. However, because most US utility companies believed these studies could be used by competitors and opponents in the regulatory arena to gain advantage, few of these studies were released to the public domain.

In 2008, the U.S. Department of Energy (DOE) funded a meta-study of outage costs, making the models to estimate outage costs publicly available and subsequently employed those models to estimate outage costs for U.S. electricity consumers [2]. Twenty-eight studies, conducted by 10 electric utilities between 1989 and 2005 representing residential and commercial and industrial (C\&I) customer groups were included in the analysis. The data was used to estimate customer damage functions expressing customer outage costs as a function of duration, time of day, consumption, business type, and other factors. The functions can be used to calculate customized outage costs for specific customers and specific durations, allowing the estimation of outage cost for the average residential and commercial customer in a given area.

The publicly available customer damage functions in this study can be employed to estimate customer outage costs with and without a smart grid investment - i.e., with current outage average duration and frequency and with reduced outage

${ }^{1}$ Two other outage cost estimation techniques have been employed: scaled macro-economic indicators (i.e., gross domestic product, wages, etc.), and market-based indicators (e.g., incremental value of reliability derived from studies of price-elasticity of demand for service offered under non-firm rates). For a detailed explanation of the different approaches, see the "Outage Cost Estimation Guidebook" [1]. 
duration and frequency. Table 1 displays estimated average electricity customer interruption costs for 2008 expressed in costs per event, costs per average $\mathrm{kW}$, costs per un-served $\mathrm{kWh}$ and costs per annual kWh. Cost estimates are provided for three customer segments and for durations ranging from less than 5 minutes (momentary) to 8 hours. They are reported for three customer classes defined as follows:

- Medium and Large C\&l (all non-residential customers with sales greater than 50,000 kWh per year);

- Small C\&l Customers (all non-residential accounts with sales less than or equal to 50,000 $\mathrm{kWh}$ per year), and;

- Residential customers.

The values in the table have been calculated using the general customer damage functions described. These customer damage functions and the results in Table 1 can be found in the report prepared for DOE by FSC and LBNL [2]. Section IV below explains how to apply these outage cost estimates to a smart grid investment opportunity.

TABLE I

ESTIMATED AVERAGE ELECTRIC CUSTOMER INTERRUPTION COSTS US 2008\$ BY CUSTOMER TYPE AND DURATION

\begin{tabular}{|c|c|c|c|c|c|}
\hline \multirow[b]{2}{*}{ Interruption Cost } & \multicolumn{5}{|c|}{ Interruption Duration } \\
\hline & Momentary & 30 minutes & 1 hour & 4 hours & 8 hours \\
\hline \multicolumn{6}{|l|}{ Medium and Large C\&I } \\
\hline Cost Per Event & $\$ 6,558$ & $\$ 9,217$ & $\$ 12,487$ & $\$ 42,506$ & $\$ 69,284$ \\
\hline Cost Per Average kW & $\$ 8.0$ & $\$ 11.3$ & $\$ 15.3$ & $\$ 52.1$ & $\$ 85.0$ \\
\hline Cost Per Un-served kWh & $\$ 96.5$ & $\$ 22.6$ & $\$ 15.3$ & $\$ 13.0$ & $\$ 10.6$ \\
\hline Cost Per Annual kWh & $9.18 \mathrm{E}-04$ & $1.29 \mathrm{E}-03$ & $1.75 \mathrm{E}-03$ & $5.95 \mathrm{E}-03$ & $9.70 \mathrm{E}-03$ \\
\hline \multicolumn{6}{|l|}{ Small C\&I } \\
\hline Cost Per Event & $\$ 293$ & $\$ 435$ & $\$ 619$ & $\$ 2,623$ & $\$ 5,195$ \\
\hline Cost Per Average kW & $\$ 133.7$ & $\$ 198.1$ & $\$ 282.0$ & $\$ 1,195.8$ & $\$ 2,368.6$ \\
\hline Cost Per Un-served kWh & $\$ 1,604.1$ & $\$ 396.3$ & $\$ 282.0$ & $\$ 298.9$ & $\$ 296.1$ \\
\hline Cost Per Annual kWh & $1.53 \mathrm{E}-02$ & $2.26 \mathrm{E}-02$ & $3.22 \mathrm{E}-02$ & $\$ 0.137$ & $\$ 0.270$ \\
\hline \multicolumn{6}{|l|}{ Residential } \\
\hline Cost Per Event & $\$ 2.1$ & $\$ 2.7$ & $\$ 3.3$ & $\$ 7.4$ & $\$ 10.6$ \\
\hline Cost Per Average kW & $\$ 1.4$ & $\$ 1.8$ & $\$ 2.2$ & $\$ 4.9$ & $\$ 6.9$ \\
\hline Cost Per Un-served kWh & $\$ 16.8$ & $\$ 3.5$ & $\$ 2.2$ & $\$ 1.2$ & $\$ 0.9$ \\
\hline Cost Per Annual kWh & $1.60 \mathrm{E}-04$ & $2.01 \mathrm{E}-04$ & $2.46 \mathrm{E}-04$ & $5.58 \mathrm{E}-04$ & $7.92 \mathrm{E}-04$ \\
\hline
\end{tabular}

These customer damage functions are able to provide estimates of the costs of interruptions of varying duration; occurring at different times of day (morning, afternoon and evening), days of week (weekends or weekdays) and season (summer and winter). They also provide estimates of interruption costs for customers of different size; and in the case of business customers, by business type (i.e., retail, utilities, construction, etc.). It is also possible to estimate costs for planned as opposed to unannounced interruptions and for customers with and without backup generation. Thus by inserting reasonable assumptions about the interruption characteristics and customers into the customer damage functions, it is possible to use them to estimate the cost of a wide range of interruptions for a wide range of customers. Then the costs can be compared with and without a reliability investment to determine the change in value of service.

\section{METHODOLOGICAL COMPARISON}

There are two key findings from these outage cost estimates that have important implications for the valuation of smart grid investments:

1. Cost per un-served kWh is substantially higher for small C\&I customers than medium and large C\&I customers.

2. A reduction in outage duration is less valuable than a reduction in outage frequency if the reduction in unserved $\mathrm{kWh}$ is equal.

Depending on the customer mix in a given area or the types of technologies in consideration, analysts may drastically under- or overestimate the value of a smart grid investment if too many simplifying assumptions are made. The following two examples compare methodologies and consider the implications of these simplifying assumptions.

\section{A. Value of Distribution Automation in California}

In a recent report prepared for the California Energy Commission (CEC) [3], the authors investigate the value of a change in SAIFI that distribution automation investments in California will provide. These investments are projected to provide a 32.7 percent decrease in SAIFI where the average outage duration is 101.9 minutes. The estimated value to the customer is $\$ 127.7$ million, of which $\$ 125.4$ million comes from the C\&I sector (see Table 2). To estimate this change in value of service, the C\&I sector was split into commercial and industrial customers and assigned different dollar values per un-served $\mathrm{kWh}$. Industrial customers were assumed to experience a loss of $\$ 25$ per un-served $\mathrm{kWh}$ and $\$ 10$ for commercial. The study that these values are based on is not reported.

However, as seen above, the more applicable C\&I grouping methodology is between small C\&I customers and medium and large C\&I customers. Therefore, we take the combined C\&I un-served $\mathrm{kWh}$ and allocate it among this alternative grouping. Based on usage data from a large California utility, we estimate that in California around 90 percent of C\&I usage is among medium and large C\&I customers and 10 percent among small C\&I customers. Using this allocation and an approximate dollar per un-served $\mathrm{kWh}$ of a 101.9 minute outage, we estimate that the change in value of service is $\$ 239.6$ million. This methodology leads to an estimated change in value of service nearly double that of the estimates in the CEC report.

TABLE II

Estimating Change in VALUE Of Service From Automated DISTRIBUTION INVESTMENTS IN CALIFORNIA - TWO METHODOLOGIES

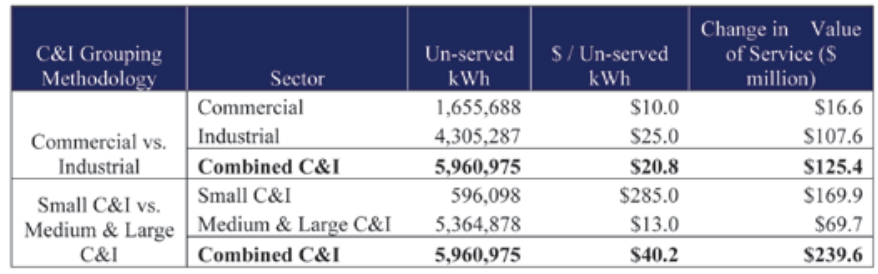

\section{B. Problems with Un-served $k$ Wh Approach}

Although we use the un-served $\mathrm{kWh}$ approach to compare differences in C\&I groupings, this methodology does not deal 
with the value of a reduction of outage duration and frequency separately. The second key finding from the aforementioned report is that a reduction in outage duration is less valuable than a reduction in outage frequency when the reduction in unserved $\mathrm{kWh}$ is equal. Therefore, the return on an investment that reduces outage frequency is greater than that of an investment that reduces outage duration, holding un-served kWh equal.

Consider this example. There are two smart grid investments in consideration. One reduces outage duration by 50 percent, and the other reduces outage frequency by 50 percent. Both reduce un-served kWh by 50 percent, but the value of each investment is quite different. Assume that in the base scenario, the average medium and large C\&I customer experiences one 1-hour outage each year. In table 1 above, this 1-hour outage costs the customer $\$ 12,487$ per year.

The investment that leads to a 50 percent reduction in outage duration will result in a situation where the average medium and large C\&I customer still experiences one outage per year, but this outage is now 30 minutes. In Table 1 above, this 30 -minute outage costs the customer $\$ 9,217$ per year. In the end, the investment that reduces outage duration by 50 percent increases value of service by $\$ 3,270$.

The investment that leads to a 50 reduction in outage frequency will result in a situation where the average outage duration is still 1-hour, but the probability of experiencing an outage is reduced by 50 percent. Therefore, a 1-hour outage still costs the customer $\$ 12,487$, but since the probability of experiencing an outage in a given year is now 50 percent as opposed to 100 percent, the outage cost to the customer is now $\$ 6,244$ per year. Although the reduction in un-served $\mathrm{kWh}$ is the same for both investments, the one that reduces outage frequency provides nearly double the value.

\section{How to Apply Outage Cost Estimates to a SMart GRID INVESTMENT OPPORTUNITY}

Now that implications of the outage cost estimates in the report [2] have been discussed, this section summarizes the methodology for correctly applying them. The estimates were based on a two-part econometric regression model. In the first part, a Probit model is estimated to determine the probability that a given outage cost is greater than zero. The second part is a generalized linear model (GLM) that estimates outage cost given that it is greater than zero.

The data used for the two-part econometric regression model came from twenty-eight surveys conducted by 10 electric utilities between 1989 and 2005. Each survey was designed to estimate outage costs for residential and small, medium and large C\&I customers. Each respondent was asked to estimate the cost of four to eight hypothetical outage scenarios of varying duration, time of year, day of week and time of day. For medium and large C\&I customers, an extensive accounting of estimated costs was done. For residential and small C\&I customers, respondents provided estimates of how much they would be willing to pay to avoid each outage scenario. The data for these twenty-eight surveys was standardized, adjusted for inflation and combined into the meta-dataset that was used to estimate the two-part model. There is substantial discussion in the methodology section of the report [2] as to why this two-part model is optimal. An extensive methodological discussion of the econometric techniques is beyond the scope of this paper.

Table 3 summarizes the regression coefficients and variables for both parts of the model. For each part of the model separately, the variable is multiplied by each coefficient and then totaled. Each of the totals is then transformed accordingly:

- For the Probit model, the sum of the cross products is run through the NORMSDIST() function in Microsoft Excel. This function always returns a value between zero and one, which is what the Probit is designed to do.

- For the GLM model, the sum of the cross products is run through the $\operatorname{EXP}()$ function in Microsoft Excel. Since the dependent variable in the GLM model was transformed by the natural log, it is necessary to exponentiate the initial GLM output.

After transforming the Probit output with NORMSDIST() and the GLM output with $\operatorname{EXP(),~the~two~numbers~are~}$ multiplied together. In the end, this provides the probability that the outage cost is greater than zero (Probit) multiplied by the level of outage cost given that it is greater than zero (GLM). The result is the expected outage cost.

TABLE III

REGRESSION COEFFICIENTS AND VARIABLES MEDIUM AND LARGE C\&I

\begin{tabular}{|l|c|c|}
\multirow{2}{*}{\multicolumn{1}{|c|}{ Variable }} & \multicolumn{2}{c|}{ Part of Model } \\
\cline { 2 - 3 } & Probit & GLM \\
\hline Duration & 0.0070 & 0.0091 \\
\hline Duration Squared & 0.0000 & 0.0000 \\
\hline Morning & 0.2002 & 0.0185 \\
\hline Afternoon & 0.3805 & 0.2801 \\
\hline Evening & -0.0197 & 0.3058 \\
\hline Summer & 0.4608 & -0.0773 \\
\hline Weekday & 0.1511 & 0.2522 \\
\hline Advanced Warning & 0.0762 & -0.0883 \\
\hline Natural Log of Annual MWh & 0.0852 & 0.4509 \\
\hline Duration X InMWh & -0.0002 & -0.0002 \\
\hline Duration Squared X InMWh & 0.0000 & 0.0000 \\
\hline Mining & 0.6854 & 0.4301 \\
\hline Construction & 0.3760 & 1.5795 \\
\hline Manufacturing & 0.5571 & 1.2892 \\
\hline Transportation, Communication \& Utilities & 0.1836 & 0.8146 \\
\hline Wholesale \& Retail Trade & 0.4550 & 0.2727 \\
\hline Finance, Insurance \& Real Estate & 0.2304 & 1.2249 \\
\hline Services & 0.1642 & 0.5216 \\
\hline Public Administration & 0.2069 & 0.6165 \\
\hline Industry Unknown & 0.1502 & 1.0763 \\
\hline Backup generation or Power conditioning & 0.0267 & 0.0804 \\
\hline Backup generation and Power conditioning & 0.2651 & 0.1272 \\
\hline Constant & -1.7060 & 4.5241 \\
\hline
\end{tabular}

Now that the calculation of the output has been discussed, it is necessary to explain each of the variables and what values to use for each:

- Duration - For the base scenario before the reliability 
investment is made, duration is the current CAIDI. After the reliability investment is made, duration is the new CAIDI. All duration values are in minutes.

- Duration squared - Squared value of duration

- Morning - Probability that an outage occurs during the morning ${ }^{4}$

- Afternoon - Probability that an outage occurs during the afternoon ${ }^{4}$

- Evening - Probability that an outage occurs during the evening $^{2}$

- Summer - Probability that an outage occurs during the summer months as opposed to non-summer months

- Weekday - Probability that an outage occurs on a weekday as opposed to the weekend

- Advanced Warning - Probability that advanced warning is given for an outage

- Natural Log of Annual MWh - Natural log of the annual MWh for the average customer

- Duration X InMWh - Duration multiplied by the natural $\log$ of annual MWh

- Duration Squared X InMWh - Duration squared multiplied by the natural log of annual MWh

- Mining through Industry Unknown - Percentage of customers that fall into each of these industry categories $^{3}$

- Backup generation or Power conditioning Percentage of customers that have backup generation or power conditioning equipment but not both ${ }^{6}$

- Backup generation and Power conditioning Percentage of customers that have backup generation and power conditioning equipment ${ }^{4}$

- Constant - The constant is simply added into the sum of the cross products from the other variables and coefficients.

In order to calculate the cost per event values in Table 1 above, the average values in Table 4 are used. The expected outage conditions simply reflect the percentage of hours in the day, week and year that each scenario is taking place. Morning is 6 out of 24 hours in the day (25\%). Afternoon and evening are 5 out of 24 hours in the day (20.8\%). Summer is 4 out of 12 months in the year (33.3\%). Weekday is 5 out of 7 days $(71.4 \%)$. It is assumed that no advanced warning is given. If an outage is more likely to occur at a certain time, these values should be adjusted.

Ideally, analysts should base the expected outage conditions off of actual data. For a summer peaking utility, the summer variable should be a larger percentage to not only reflect the 4

\footnotetext{
2 The base scenario in the model is the probability that an outage occurs at night. This scenario is omitted from the regressions. The sum of the probabilities that an outage occurs during the morning, afternoon, evening and night must be 1 .

3 The base industry category in the model is Agriculture, Forestry and Fishing. This industry category must be accounted for so that all 10 industry categories total 100 percent.

4 The base category is no backup generation or power conditioning equipment. The three categories must total 100 percent.
}

months of summer in the year, but the fact that outages are more likely to occur during the summer. Historical data can be used to determine when outages are likely to occur, and the expected outage conditions can reflect those numbers. This method will provide the most accurate estimates for a given area.

The average customer characteristics should also be based off of actual data in a given area. The average annual $\mathrm{MWh}$ for a medium and large C\&I customer is relatively straightforward to determine. The percentages for each industry reflect the industry mix in a given area. Finally, at least a rough estimate of the two backup generation and power conditioning variables should be used.

TABLE IV

INPUTS FOR OUTAGE COST REGRESSION

\begin{tabular}{|c|c|}
\hline Variable & Average Value \\
\hline \multicolumn{2}{|l|}{ Expected Outage Conditions } \\
\hline Morning $(6 \mathrm{am}$ to $12 \mathrm{pm})$ & $25.0 \%$ \\
\hline Afternoon (12pm to $5 \mathrm{pm})$ & $20.8 \%$ \\
\hline Evening $(5 \mathrm{pm}$ to $10 \mathrm{pm})$ & $20.8 \%$ \\
\hline Summer (June to September) & $33.3 \%$ \\
\hline Weekday & $71.4 \%$ \\
\hline Advanced Warning & $0.0 \%$ \\
\hline \multicolumn{2}{|l|}{ Average Customer Characteristics } \\
\hline Annual MWh & 7140.5 \\
\hline Mining & $1.4 \%$ \\
\hline Construction & $0.9 \%$ \\
\hline Manufacturing & $28.6 \%$ \\
\hline Transportation, Communication \& Utilities & $7.2 \%$ \\
\hline Wholesale \& Retail Trade & $25.0 \%$ \\
\hline Finance, Insurance \& Real Estate & $3.8 \%$ \\
\hline Services & $25.2 \%$ \\
\hline Public Administration & $1.8 \%$ \\
\hline Industry Unknown & $4.7 \%$ \\
\hline Backup generation or Power conditioning & $37.2 \%$ \\
\hline Backup generation and Power conditioning & $8.4 \%$ \\
\hline
\end{tabular}

An important step is to adjust the estimates for inflation. When the regressions were estimated, 2008 dollar values were used. This needs to be taken into consideration in the years to come. These 2008 dollar values can be adjusted by using readily available inflation indicators such as the Consumer Price Index or the GDP Deflator.

Once the outage cost has been calculated under the current CAIDI and the new CAIDI, the change in SAIFI needs to be accounted for also. The change in value of service is the expected annual outage cost under the current CAIDI and SAIFI minus the expected annual outage cost under the new CAIDI and SAIFI. This value must be multiplied by the total number of medium and large C\&I customers to estimate the aggregate number. Since this is an annual estimate of the value of service, it needs to be multiplied by the number of years of useful life of the investment. A discount rate for future payments also needs to be taken into account.

Once the change in value of service has been calculated for 
the medium and large C\&I sector, the same must be done for the small C\&I sector and the residential sector using the same methodology, but different models from the report [2]. The results will provide the most accurate estimate of the change in value of service that a given smart grid investment provides.

\section{CONCLUSION}

A recent study has shown that a change in the methodology for estimating the value of reliability improvements is needed. Nonetheless, many analysts continue to use other methodologies that more heavily rely on simplifying assumptions. Problems with these simplifying assumptions commonly arise in two situations:

1. When the C\&I categorization is done incorrectly, or;

2. When un-served kWh is measured the same whether it results from outage duration or frequency.

This paper has shown that if these two issues are not accounted for, analysts may drastically under- or overestimate the change in value of service that results from a smart grid investment. By then explaining how to use the most current estimates of outage costs, it is hopeful that more analysts will employ this methodology.

\section{REFERENCES}

[1] M. J. Sullivan, and D. M. Keane. "Outage Cost Estimation Guidebook," EPRI Research Project 2878-04 Final Report, December 1995.

[2] M. J. Sullivan, M. G. Mercurio, and J. A. Schellenberg. "Estimated Value of Service Reliability for Electric Utility Customers in the United States," LBNL Research Project Final Report, June 2009.

[3] S. Blazewicz, G. Shlatz, F. Small, S. Tobias, and J. Bean. "The Value of Distribution Automation,” California Energy Commission Research Project CEC-500-2007-103 Final Report, March 2009.

\section{BIOGRAPHIES}

Dr. Michael Sullivan is chairman and co-founder of Freeman, Sullivan \& Co. He has published a number of works regarding customer response to service reliability issues. He is also a recognized authority in risk analysis and liability evaluation. He has extensive experience as an expert witness on issues of statistical analysis of product failure and construction defects, economic risks of claims and settlements, and economic models of future liability. In addition, Dr. Sullivan has pioneered the application of statistical and economic analysis disciplines, as well as the methodologies of survey and sample design to construction defect defense and to the management of product performance liabilities in the context of class action settlements and judgments.

Dr. Matthew Mercurio specializes in applied statistical analysis and econometric modeling. He has applied these skills to both public and private litigation, as well as other legal matters. Dr. Mercurio's skills and experience in applied statistical analysis include: analysis of longitudinal (panel) data, limited dependent and latent variables, analysis of time series data, including ARIMA modeling, event studies, forecasting, and simulation, and aurvey design and sampling analysis. Dr. Mercurio holds a Ph.D. in economics from Princeton University and a BA in Economics and Mathematics from Boston University.
Josh Schellenberg has been instrumental in developing impact evaluations for demand response programs at multiple utilities. In 2009, he evaluated California's Base Interruptible Program, which is one of the largest demand response resources in the United States with nearly $1 \mathrm{GW}$ of load reduction capacity and participation from three large IOUs. He also played a key role in the econometric modeling for the expost evaluation of Pacific Gas \& Electric's SmartRate program, which was one of the largest deployments of CPP in the country. Concurrently, Mr. Schellenberg has worked on each of the annual evaluations of Southern California Edison's demand response portfolio since 2008.

Joseph Eto is a staff scientist in the Environmental Energy Technologies Division of the Lawrence Berkeley National Laboratory. His principal responsibility is management of the Consortium for Electric Reliability Technology Solutions, which is coordinating multi-institution, public-interest research to maintain and enhance the reliability of power systems during the transition to restructured electricity markets. Mr. Eto has authored over 150 publications on electricity policy, electricity reliability, transmission planning, cost-allocation, demand response, distributed energy resources, utility integrated resource planning, demand-side management, and building energy-efficiency technologies and markets. 